\title{
Clinical characteristics of patients with large and deep soft tissue sarcomas
}

\author{
TOMOKI NAKAMURA, AKIHIKO MATSUMINE, TAKAO MATSUBARA, \\ KUNIHIRO ASANUMA, ASTUMASA UCHIDA and AKIHIRO SUDO
}

\author{
Department of Orthopaedic Surgery, Mie University Hospital, Tsu, Mie 514-8507, Japan
}

Received August 11, 2014; Accepted April 29, 2015

DOI: $10.3892 / \mathrm{ol} .2015 .3289$

\begin{abstract}
In the present study, the incidence of surgical wound complications in patients with large and deep high-grade soft tissue sarcoma (STS) was investigated. In addition, the disease-specific and event-free survival rates were examined in these patients. In total, 30 adult patients with primary high-grade deep STS with a size of $10 \mathrm{~cm}$ or greater were treated in the current study. Of the 30 patients, 16 (53\%) developed a total of 19 wound complications. In addition, 10 cases of wound dehiscence, 5 cases of infections, 3 cases of hematomas and 1 case of lymphorrhea were reported, while 3 patients with postoperative infections underwent additional surgical treatment. Longer surgery duration was associated with wound complications $(\mathrm{P}=0.04)$. The disease-specific survival was $58.5 \%$ at 3 years and $46.1 \%$ at 5 years after treatment. Furthermore, local recurrence was detected in 13 patients and distant metastasis was identified in 17 patients as the first relapse. The 3- and 5-year event-free survival rates were 24.4 and $16.3 \%$, respectively. In conclusion, patients with large and high-grade, deep STSs presented a high complication rate; therefore, careful wound care is required in these patients. Furthermore, it should be also taken into account that these patients have a greater risk of tumor-associated events and mortality.
\end{abstract}

\section{Introduction}

Soft tissue sarcomas (STSs) are rare tumors representing $<1 \%$ of all adult malignancies and accounting for at least 11,410 new diagnoses each year (1). The most common primary sites are the extremities, followed by the trunk, internal trunk (such as the retroperitonium), pelvic cavity, intra-abdominal region, and the head and neck (1). Detection of STS is easier in cases where the tumor is superficial. However, the early diagnosis of a deep STS may be difficult, since a number of these tumors

Correspondence to: Dr Tomoki Nakamura, Department of Orthopaedic Surgery, Mie University Hospital, 2-174 Edobashi, Tsu, Mie 514-8507, Japan

E-mail: tomoki66@clin.medic.mie-u.ac.jp

Key words: soft tissue sarcoma, wound complication, survival present as a painless mass $(2,3)$. As a result, numerous STSs are diagnosed at a late stage and have already reached a large size at the time of presentation. High-grade STS resection with an adequate wide margin reportedly inhibits local tumor recurrence and improves patients prognosis compared with a marginal or intra-lesional margin (4). Patients with larger tumors are in greater risk of requiring an amputation as the primary treatment strategy and have a higher incidence of postoperative surgical wound complications, as well as mortality and tumor recurrence (2,3,5-11). In addition, patients with high-grade STS may have a higher wound complication rate. This is due to a larger incision, large defect of soft tissue, which is a result of a wider margin of tissue requiring removal, prolonged surgery duration and other factors (5-9). In addition to tumor size, tumor depth and tumor histological grade have also been demonstrated to be predictive factor for survival $(10,11)$. Therefore, patients with high-grade, deep and large STS must be carefully treated and followed-up.

Only a limited number of studies exist that report detailed surgical and clinical outcome in such patients $(2,6)$. Therefore, the aim of the present study was to elucidate the incidence of surgical complications and the survival in patients with large and deep high-grade STS. Furthermore, the disease-specific and event-free survival rates were examined in these patients.

\section{Patients and methods}

Patients. Between January 1999 and March 2013, a total of 30 adult patients suffering from primary high-grade deep STS with a size of $10 \mathrm{~cm}$ or greater were treated at the Mie University Hospital (Tsu, Japan). A deep tumor was defined as a tumor with any of following characteristics: Located exclusively beneath the superficial fascia; superficial to the fascia, with invasion of or through the fascia; or both superficial to and beneath the fascia (12). All 30 patients underwent surgical resection of the tumor. Patients that presented with metastases and/or local recurrence at the diagnosis, as well as patients with retroperitoneal sarcomas, were excluded from the present study. The pre-treatment work-up included brain, lung, abdomen and pelvic computed tomography scans with and without the use of a contrast medium. The histopathological diagnosis and tumor grade were determined using the French Federation of Cancer Centers Sarcoma Group (FNCLCC) grading system (13) for all the patients; the grading was reviewed and confirmed by 
two independent pathologists. In addition, the surgical margin was microscopically evaluated. The study was approved by the Ethics Committee of Mie University Hospital. Written informed consent was obtained from the patients.

Statistical analysis. Statistical associations of the clinicopathological factors were evaluated using the Mann-Whitney U-test for quantitative data, and the $\chi^{2}$ test or Fisher's exact test for qualitative data. The duration of disease-specific or event-free survival was defined as the interval between the date of the initial treatment of the primary tumor and the date of mortality, local recurrence or metastasis. Survival curves were constructed using the Kaplan-Meier method. The log-rank test was used to compare the survival and event rates. Statistical analyses were performed using the Stat View version 5.0 software (SAS Institute, Cary, NC, USA). A value of $\mathrm{P}<0.05$ was considered to indicate a statistically significant difference.

\section{Results}

Patient, tumor and treatment characteristics. Table I reports the characteristics and treatments of patients included in the present study. The mean age at diagnosis was 62 years (range, 24-86 years), while 20 male and 10 female patients were included. The mean interval between the onset of symptoms and the histological diagnosis was 1-50 months, with a median period of 5.5 months. In total, 11 patients experienced a delay in symptom onset of $>6$ months. The main symptoms resulting in the patients consulting the Mie University Hospital were as follows: Increased size of the mass $(n=12)$; awareness of the mass $(n=7)$; and pain $(n=13)$. In addition, 2 patients experienced a combination of two clinical symptoms, which prompted them to consult our hospital. The mean tumor size at diagnosis was $15.1 \mathrm{~cm}$ (range, 10-30 cm). According to the FNCLCC grading system, 19 patients had grade 3 sarcomas and 11 patients had grade 2 tumors. Furthermore, the tumors were histologically classified as 9 cases of malignant fibrous histiocytoma/undifferentiated pleomorphic sarcoma, 5 cases of myxoid liposarcoma, 4 cases of malignant peripheral nerve sheath tumor, 3 cases of leiomyosarcoma, 2 cases of myxofibrosarcoma, 2 cases of malignant granular cell tumor, 2 cases of fibrosarcoma, and 1 case each of extra-skeletal chondrosarcoma, dedifferentiated liposarcoma and epithelioid sarcoma. The primary tumor sites included the thighs $(n=18)$, buttocks $(n=7)$, chest wall $(n=2)$ and other sites $(n=3)$. Finally, the mean follow-up period after the date of the initial treatment was 40 months (range, 6-148 months).

Surgical treatment and wound complications. In total, 28 of the 30 patients underwent limb salvage surgery and 2 patients underwent amputation of the primary sarcoma (Table I). The surgical complications experienced by the patients are listed in Table II, while Table III reports the association between the complications and patient characteristics.

Of the 30 patients included in the present study, 16 (53\%) developed a total of 19 wound complications (Table II). There were 10 cases of wound dehiscence, 5 cases of infections, 3 cases of hematomas and 1 case of lymphorrhea. No patients succumbed to these surgical complications. Additional surgical treatment was performed in 3 patients with postoperative
Table I. Patient characteristics.

Characteristic

Patients, $\mathrm{n}=30$

\begin{tabular}{lc}
\hline Age (years) & \\
Mean & 62 \\
Range & $24-86$ \\
Gender (n) & \\
Male & 20 \\
Female & 10 \\
Tumor size (cm) & \\
Mean & 15.1 \\
Range & $10-30$ \\
Surgical procedure (n) & \\
Amputation & 2 \\
Limb salvage surgery & 28 \\
$\quad$ Resection & 20 \\
Resection and prosthesis & 5 \\
Resection and mesh & 1 \\
Resection and intramedullary nail & 3 \\
Surgical margin (n) & \\
Positive & \\
Negative & 23 \\
\hline
\end{tabular}

Table II. Type of surgical wound complication.

Surgical complication

Patients $(n)^{\mathrm{a}}$

$\begin{array}{lc}\text { Infection } & 5 \\ \text { Hematoma } & 3 \\ \text { Wound dehiscence } & 10 \\ \text { Lymphorrhea } & 1 \\ \text { None } & 14\end{array}$

${ }^{a}$ Certain patients $(n=3)$ developed two complications each: One patient developed infection and wound dehiscence; one patient presented hematoma and wound dehiscence; the other patient presented lymphorrhea and wound dehiscence.

infections, while 2 patients with postoperative hematomas underwent puncture.

Reconstruction subsequent to tumor resection was required in 6 patients (Table III), whereas prosthesis following tumor resection, including a part of the femur, was required in 5 patients due to suspected bone invasion based on the pre-treatment examination. For skeletal reconstruction after tumor resection of normal muscle and rib attached to the tumor at the chest wall, a prosthetic mesh was used in 1 case. The mean intraoperative blood loss was 1,006 $\mathrm{ml}$ (range, 68-3,170 $\mathrm{ml}$ ) and intraoperative blood transfusion was required in 12 patients (40\%). The mean operative time was 224 min (range, 78-689 min). Histologically, a positive surgical margin was observed in 7 patients, while a negative surgical margin was identified in 23 patients (Table I). 
Table III. Association between wound complications and patient characteristics.

\begin{tabular}{|c|c|c|c|}
\hline \multirow[b]{2}{*}{ Variables } & \multicolumn{2}{|c|}{ Complication } & \multirow[b]{2}{*}{ P-value ${ }^{a}$} \\
\hline & Yes & No & \\
\hline \multicolumn{4}{|l|}{ Age (years) } \\
\hline Mean age & 65 & 58 & 0.15 \\
\hline \multicolumn{4}{|l|}{ Gender (n) } \\
\hline Male & 11 & 9 & 0.80 \\
\hline Female & 5 & 5 & \\
\hline \multicolumn{4}{|c|}{ Intraoperative blood loss (ml) } \\
\hline Mean & 1,310 & 659 & 0.07 \\
\hline \multicolumn{4}{|c|}{ Surgery duration (min) } \\
\hline Mean & 264 & 179 & 0.04 \\
\hline \multicolumn{4}{|c|}{ Reconstruction after surgery (n) } \\
\hline Yes & 3 & 4 & 0.67 \\
\hline \multicolumn{4}{|c|}{ Tumor resection (n) } \\
\hline No & 13 & 10 & \\
\hline \multicolumn{4}{|c|}{ Neo-adjuvant Cx (n) } \\
\hline Yes & 4 & 6 & 0.44 \\
\hline No & 12 & 8 & \\
\hline \multicolumn{4}{|c|}{ Radiation therapy (n) } \\
\hline Yes & 4 & 12 & \\
\hline No & 2 & 12 & 0.66 \\
\hline \multicolumn{4}{|c|}{ Tumor grade (n) } \\
\hline Grade 2 & 7 & 4 & \\
\hline Grade 3 & 9 & 10 & 0.39 \\
\hline
\end{tabular}

${ }^{a} \mathrm{P}<0.05$ indicate that the variable is associated with increased incidence of complications. $\mathrm{Cx}$, chemotherapy.

To prevent a fracture following brachytherapy, an intramedullary nail was inserted at the femur in 3 patients. In addition, 10 of the 30 patients received neoadjuvant and/or adjuvant chemotherapy, 4 underwent brachytherapy and 2 received postoperative radiotherapy.

The results of the statistical analysis indicated that longer surgery duration was associated with wound complications $(\mathrm{P}=0.04$, Mann-Whitney U test). However, blood loss $(\mathrm{P}=0.07$, Mann-Whitney $U$ test) and administration of adjuvant therapy (chemotherapy or radiotherapy; $\mathrm{P}=0.70, \chi^{2}$ test) was not associated with wound complications. By contrast, the 2 patients treated with amputation experienced no complications.

Clinical outcome in patients with high-grade STS. Of the 30 patients included in the present study, 12 were alive and disease-free in March 2014 (last review), while 3 patients were alive with disease. However, 13 patients succumbed to the disease and 2 due to other causes; the average survival time after the treatment in these 13 patients was 22.8 months (range, 6-59 months) The disease-specific survival was $58.5 \%$ at 3 years and $46.1 \%$ at 5 years after treatment (Fig. 1). Local recurrence was detected in 13 patients, and distant metastasis was identified in 17 patients as the first relapse. The 3 - and

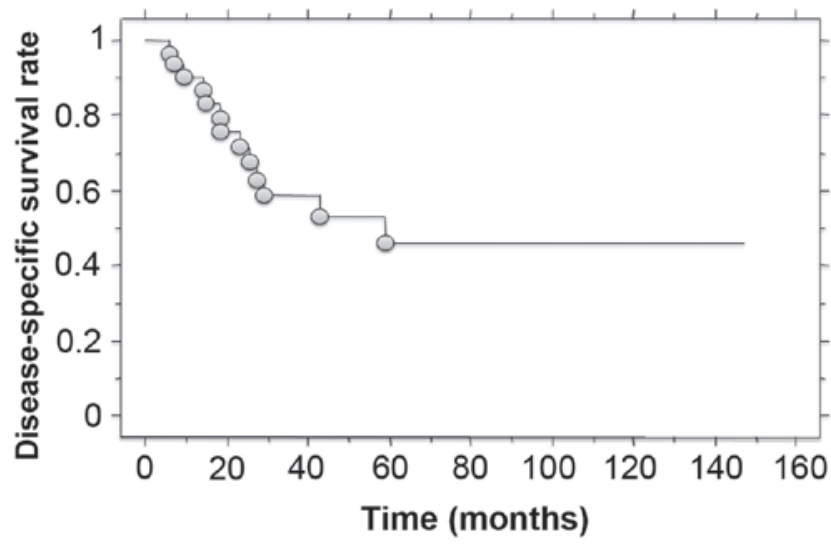

Figure 1. Kaplan-Meier curve demonstrating the overall disease-specific survival of the 30 STS patients.

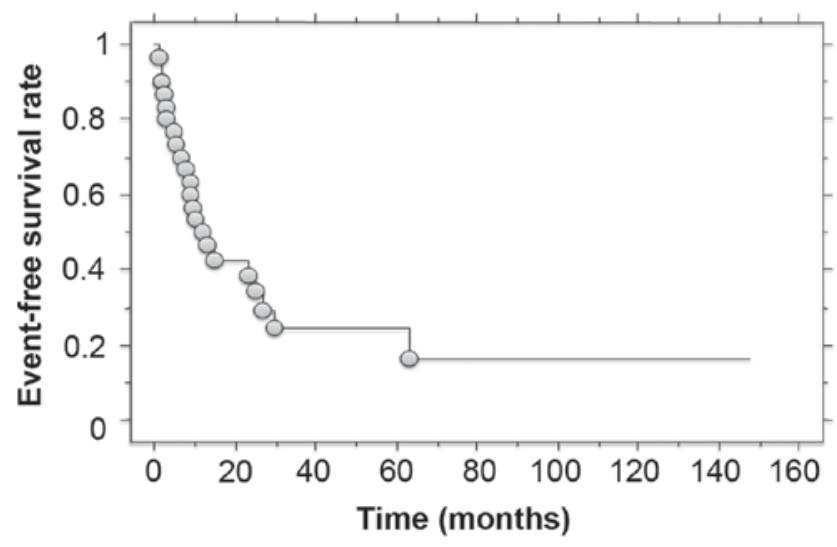

Figure 2. Kaplan-Meier curve demonstrating the event-free survival of the 30 STS patients.

5-year event-free survival rates were 24.4 and $16.3 \%$, respectively (Fig. 2). Of the 28 patients treated with limb salvage surgery, 3 patients eventually underwent amputation due to local recurrence. The log-rank test revealed no statistically significant association between disease-specific or event-free survival, and the following factors: patient age and gender, tumor histological grade and administration of adjuvant therapy.

\section{Discussion}

The early diagnosis of deep STS may be difficult, particularly in tumors located at the thigh or buttock due to the muscle volume of these areas $(2,3)$. Furthermore, numerous of these tumors present as a painless mass $(2,3)$. Surgery continues to play an important role in the treatment of soft tissue sarcoma, which can involve local resection and subsequent reconstruction of the resultant deficit, or amputation. However, particularly in large tumors, surgery for STS is associated with several risk factors of surgical wound complications, including a large defect following tumor resection, blood loss, the administration of adjuvant therapy and the requirement for prosthetic reconstruction following tumor resection, such as bone reconstruction (5-9). In the current study, 53\% of patients with large and deep, high-grade STS presented 
wound complications, and the surgery duration was found to be significantly associated with the incidence of these complications. Although a high incidence of wound complications was observed, there were no fatal complications among the investigated cases. Therefore, taking into account the risk of complications and subsequent treatments, a large number of these patients were able to recover.

While multiple factors affect the survival following STS treatment, the tumor size, depth and histological grade are certainly the most important factors $(2,3,10,11)$. Weitz et al (11) reported that the 5-year disease-specific survival rate was $51 \%$ for high-risk patients (high-grade, deep tumors, with a size of $>10 \mathrm{~cm})$. In the cases examined in the present study, the disease-specific survival rate was $58.5 \%$ at 3 years and $46.1 \%$ at 5 years after treatment. These results suggest that these tumors have a high potential of local recurrence and metastases. In fact, in present study, 43 and $57 \%$ of patients developed local recurrence and metastases, respectively. Therefore, careful follow-up should be performed to detect early relapse for these patients. Furthermore, since limb salvage surgery results in several complications and risk of recurrence, it must be considered whether amputation may be a better strategy. No predictive factors for survival and event incidence were detected in the current cases, possibly due to the high risk of oncological event in the patient cohort.

However, the current study presents certain limitations. First, the presence of systemic diseases may be associated with a higher rate of wound complications. Clinical factors, including medical comorbidities, obesity, smoking and the patients' immunocompetence, were not considered in the study due to the lack of information. Furthermore, the retrospective nature of the study is another limitation.

In conclusion, surgical wound complications occurred in $53 \%$ of patients with high-grade STS in the present study. Therefore, careful wound care is required in these patients. Furthermore, the fact that these patients are at a greater risk of tumor-associated events and mortality should be considered during treatment.

\section{References}

1. Kneisl JS, Coleman MM and Raut CP: Outcome in the management of adult soft tissue sarcomas. J Surg Oncol 110: 527-538, 2014.

2. Grimer RJ: Size matters for sarcomas! Ann R Coll Surg Engl 88: 519-524, 2006

3. Nakamura T, Matsumine A, Matsubara T, et al: The symptom-to diagnosis delay in soft tissue sarcoma influence the overall survival and the development of distant metastasis. J Surg Oncol 104: 771-775, 2011.

4. Matsubara T, Kusuzaki K, Matsumine A, Nakamura T and Sudo A: Can a less radical surgery using photodynamic therapy with acridine orange be equal to a wide-margin resection? Clin Orthop Relat Res 471: 792-802, 2013.

5. Saddegh MK and Bauer HC: Wound complication in surgery of soft tissue sarcoma. Analysis of 103 consecutive patients managed without adjuvant therapy. Clin Orthop Relat Res 289: 247-253, 1993.

6. Aded R and Younge D: Surgical management of very large musculoskeletal sarcomas. Ann N Y Acad Sci 1138: 77-83, 2008.

7. Geller DS, Hornicek FJ, Mankin HJ and Raskin KA: Soft tissue sarcoma resection volume associated with wound-healing complications. Clin Orthop Relat Res 459: 182-185, 2007.

8. Schwartz A, Rebecca A, Smith A, et al: Risk factors for significant wound complications following wide resection of extremity soft tissue sarcomas. Clin Orthop Relat Res 471: 3612-3617, 2013.

9. Peat BG, Bell RS, Davis A, et al: Wound-healing complications after soft-tissue sarcoma surgery. Plast Reconstr Surg 93: 980-987, 1994.

10. Pisters PW, Leung DH, Woodruff J, Shi W and Brennan MF: Analysis of prognostic factors in 1,041 patients with localized soft tissue sarcomas of the extremities. J Clin Oncol 14: 1679-1689, 1996

11. Weitz J, Antonescu CR and Brennan MF: Localized extremity soft tissue sarcoma: Improved knowledge with unchanged survival over time. J Clin Oncol 21: 2719-2725, 2003.

12. Edge SB, Byrd DR, Compton CC et al (eds): Soft tissue sarcoma. In: AJCC Cancer Staging Manual. 7th edition. Springer, New York, NY, pp345-355, 2010.

13. Trojani M, Contesso G, Coindre JM, et al: Soft-tissue sarcomas of adults; study of pathological prognostic variables and definition of a histopathological grading system. Int J Cancer 33: 37-42, 1984. 